\title{
Einsatz eines Geographischen Informationssystems zur Untersuchung der Verbreitung einer Rinderkrankheit in Afrika
}

\begin{abstract}
East Coast fever (ECF) kills hundreds of thousands of cattle in Eastern, Central and Southern Africa each year. To focus safe and cost-effective disease control programmes, the distribution of cattle and buffalo, the main hosts of the disease, as well as the present and potential distribution of the tick which carries ECF have to be considered. Maps of the relevant parameters describing the present situation of ECF were digitized by the United Nations Environment Programme in Nairobi in conjunction with the International Laboratory for Research On Animal Disease. Potentially suitable areas for the tick were determined by interpolating and modeling data from meteorological stations - using altitude information to improve the results of the interpolation - and incorporating satellite derived vegetation data. By comparing the map of potential areas with the present distribution of ECF, tick and host, critical regions may be identified, where the disease is not yet present, but into which it could spread.
\end{abstract}

\section{Einleitung}

GRID (Global Resource Information Database), ein Unterprogramm der UNO-Umweltbehörde UNEP (United Nations Environment Programme) in Nairobi, Kenia, entwickelte sich seit 1985 zu einem wichtigen Zentrum für Anwendungen von Geographischen Informationssystemen (GIS) in Afrika. Mit dem Aufbau von global, kontinental oder national, für den Umweltschutz relevanten Datenbanken soll eine tragfähige Nutzung der beschränkten natürlichen Ressourcen unterstützt und ihr Schutz ermöglicht werden. GRID hat es sich ferner zum Ziel gesetzt, die GIS-Technologie in Entwicklungsländern einzuführen, lokale Wissenschafter und Wissenschafterinnen zu schulen sowie andere internationale Organisationen in diesem Bereich zu unterstützen.

So arbeitet GRID seit 1987 mit der ebenfalls in Nairobi ansässigen ILRAD (International Laboratory For Research On Animal Diseases) zusammen an einer Studie über die räumlichen Aspekte des ostafrikanischen Küstenfiebers. Die tatsächliche und potentielle Ausbreitung dieser gefürchteten Rinderkrankheit wurde für den ganzen afrikanischen Kontinenten mit einem GIS' untersucht.

Die noch heute andauernde interdisziplinäre Zusammenarbeit hat sich äußerst fruchtbar erwiesen, gewannen doch beide Seiten einen vertieften Einblick in die Probleme und Arbeitsmethoden der anderen Organisation. Maßgeblich zum guten Gelingen dieses Projektes trug bei, daß klare Problemstellungen und das veterinärmedizinische Fachwissen der ILRAD-Fachleute immer im Vordergrund standen. GRID konnte sich voll auf eine angepaßte, technische Unterstützung konzentrieren und das GIS als Werkzeug einsetzen.

Einen Überblick über die aus dieser Zusammenarbeit resultierende GIS-Anwendung will der vorliegende Artikel vermitteln. Teile davon wurden bereits an der ARC/ INFO European User Conference im Oktober 1989 in Rom präsentiert, wo vor allem die technischen Aspekte zur Sprache kamen. ${ }^{2}$ Detaillierte Angaben über die veterinär-medizinischen Aspekte der Krankheit, die verwendete Literatur und Datenquellen sind in Publikationen von ILRAD zu finden. ${ }^{3}$

\section{Das ostafrikanische Küstenfieber}

Obwohl keine umfassenden Statistiken existieren, steht fest, daß das ostafrikanische Küstenfieber jährlich für den Tod von Hunderttausenden von Rindern im östlichen, zentralen und südlichen Afrika verantwortlich ist. Die Krankheit wird durch den Parasiten Theileria parva verursacht und durch einen Vektoren, die braune Zecke Rhipicephalus appendiculatus, übertragen. Die häufig wegen ihren höheren Milch- und Fleischerträgen gehaltenen nicht-afrikanischen Viehrassen sind am stärksten küstenfiebergefährdet, aber auch einheimische Rinder können befallen werden.

Der afrikanische Büffel (Synercus caffer), obwohl selbst nicht vom Küstenfieber betroffen, ist nach dem Rindvieh der zweitwichtigste Wirt für die braune Zecke und gefährdet Viehbestände in all denjenigen Gebieten, wo er gemeinsam mit ihnen vorkommt (Abbildung 1).

Die gewaltigen, durch das Küstenfieber verursachten Verluste rufen nach seiner Bekämpfung, wobei die vollständige Ausrottung sehr teuer und mit schwer abschätzbaren Nebenwirkungen verbunden wäre. Lokal kann das ostafrikanische Küstenfieber aber bereits heute durch ein regelmäßiges Eintauchen der Rinder in Bäder mit Milbenbekämpfungsmitteln (cattle dipping) kontrolliert werden. Diese am weitesten verbreitete Methode zur Abtötung der Zecken ist ebenso wie neue Immunisierungsund Heilungsmethoden sehr teuer.

Kaspar Kundert, dipl. Geograph, UNEP/GRID, 6 rue de la Gabelle, 1227 Carouge 


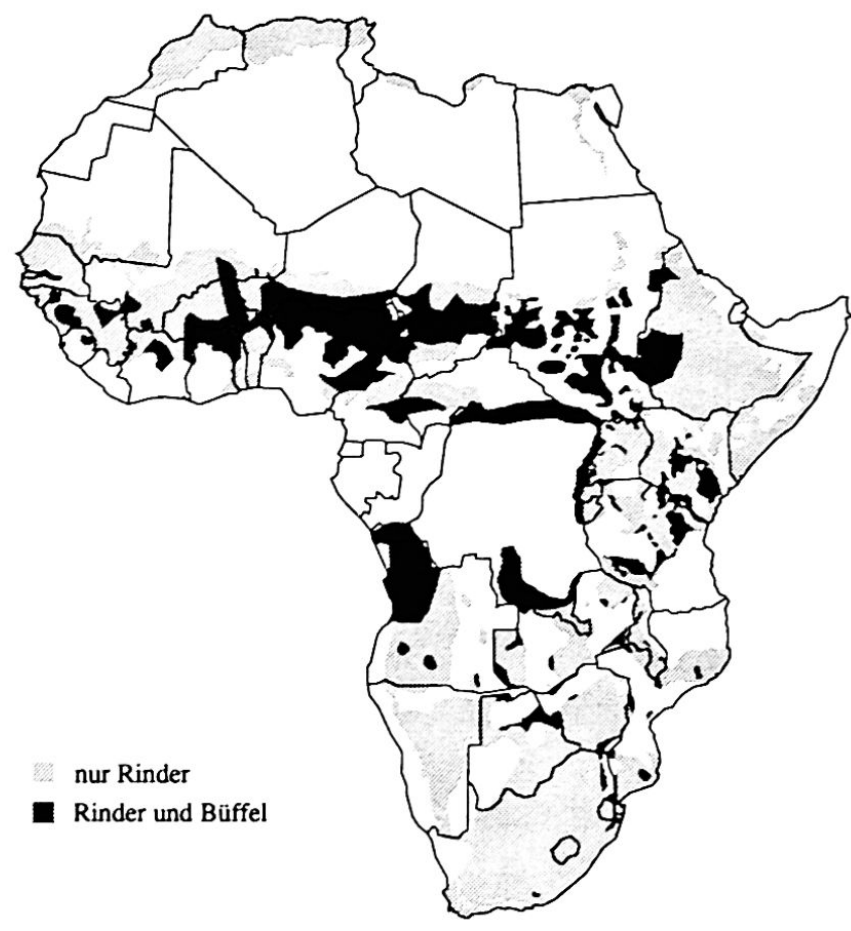

Abb. 1: Rinder- und Büffelverteilung

Um die knappen Mittel optimal für eine sichere Kontrolle des ostafrikanischen Küstenfiebers einzusetzen, ist es nötig, die momentane und potentielle Verteilung der braunen Zecke sowie die Verteilung ihrer beiden wichtigsten Wirte, der Rinder und Büffel, möglichst genau zu kennen.

\section{Die epidemiologischen Parameter des ostafrikanischen Küstenfiebers; Datenerhebung}

\subsection{Einfiihrung}

Die Veterinäre von ILRAD legten fest, welche Parameter für die räumliche Ausbreitung des Küstenfiebers bestimmend sind. Zusammen mit GRID wurden dann diejenigen ausgewählt, die digital erfaßt werden sollten, wobei es zu berücksichtigen galt, da $ß$ viele Daten nicht oder nur mit sehr geringer räumlicher Auflösung für den ganzen afrikanischen Kontinent erhältlich waren. Alle ausgewählten Parameter lassen sich einer der folgenden Kategorien zuordnen:

- Krankheitsspezifische Parameter

- Wirtspezifische Parameter

- Klima

- Topographie

- Vegetation

\subsection{Krankheits- und wirtspezifische Parameter}

Für die krankheits- und wirtspezifischen Parameter wurde auf Karten in den verschiedensten Maßstäben und
Projektionen sowie auf Tabellen und Berichten von ILRAD zurückgegriffen. Alle räumlich lokalisierbare Information, beispielweise auch in Berichten mit ihren Koordinaten erwähnte Fundstelle der braunen Zecke, wurde digitalisiert. ${ }^{+}$

Von allen digitalisierten Daten wurden für jedes betroffene Land auf einem kleinen Plotter Karten erstellt, welche an dort ansässige Küstenfieber-Fachleute versandt wurden. Ihre Kommentare waren oft die einzige Möglichkeit, einen Aufschluß über die Qualität der aufgenommenen Daten zu erhalten. Vorgeschlagene Korrekturen wurden überprüft und in die Datenbank aufgenommen.

Diese enthält jetzt Dateien über die bekannten Verteilungen des ostafrikanischen Küstenfiebers (Abbildung 2), der braunen Zecke, der Büffel und der Rinder. Für die Datei der Rinderbestände diente die OAU-Karte über die Rinderverteilung in Afrika ${ }^{5}$ als Grundlage, wurde aber, wo erhältlich, mit detaillierteren nationalen Daten ergänzt. Aus diesem Vorgehen resultierte eine Datei von sehr heterogener Qualität und Zuverlässigkeit. Trotzdem verwendet wurde sie, weil höchstens für kleinere Gebiete, nicht aber für den ganzen afrikanischen Kontinent bessere Alternativen zur Verfügung stehen.

Die anläßlich eines früheren GRID-Projektes ${ }^{6}$ erhobenen und in der GRID-Datenbank für Afrika gespeicherten Dateien über die geschützten Gebiete (Tierparks) und die Verteilung der Tsetse-Fliege (glossina spp.) wurden für Konsistenzkontrollen der digitalisierten Rinderverteilungskarte verwendet, weil weder in gut geschützten Tierparks noch in stark von Tsetse-Fliegen befallenen Gebieten hohe Viehdichten vorkommen sollten.

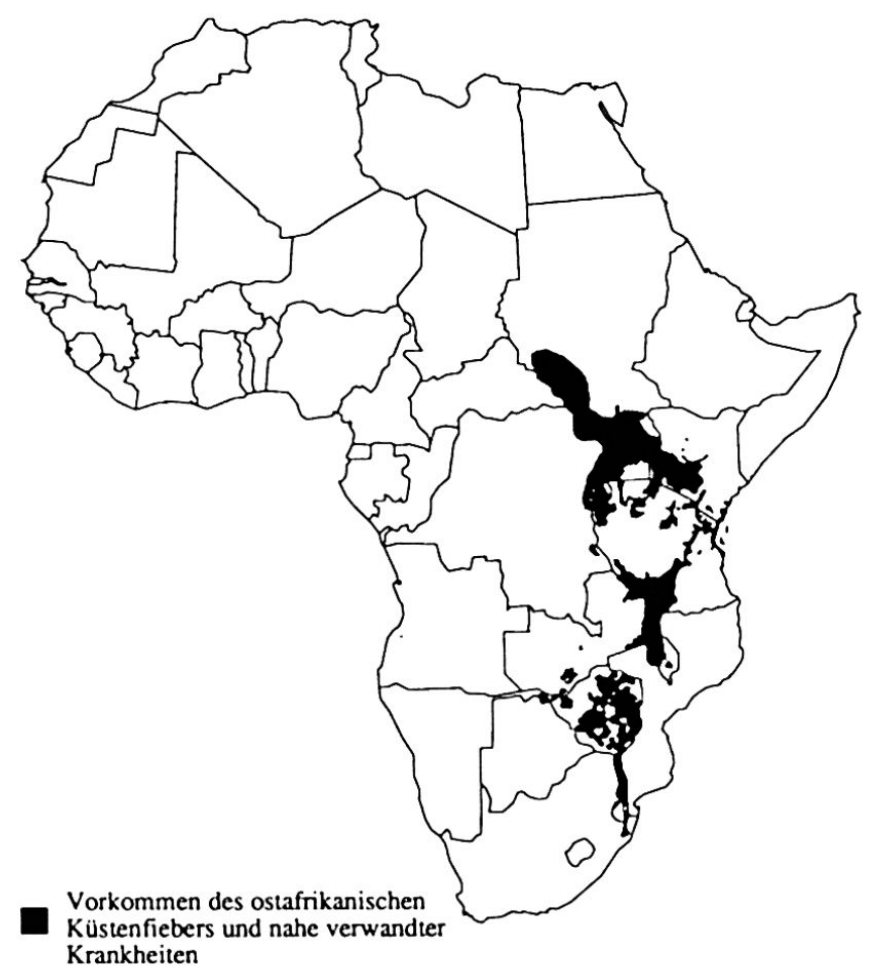

Abb. 2: Ostafrikanisches Küstenfieber 


\subsection{Klima und Topographie}

Die braune Zecke ist für ihre Entwicklung, Fortpflanzung und ihr Überleben auf ein ganz bestimmtes Klima angewiesen, welches bestimmt ist durch die jeweiligen durchschnittlichen Monatswerte der Minimum- und Maximumtemperaturen (Extremtemperaturen), des Niederschlages und der Evaporation.

Schnell stellte sich heraus, daß die räumliche Auflösung der für diese Parameter existierenden Klimakarten nicht genügt für die angestrebte Untersuchung, wo auch kleinere Gebiete mit eigenständigen klimatischen Bedingungen interessierten. So sind beispielweise die kühlen und feuchten Berge in den nordkenianischen Trockengebieten eigentliche Oasen für Mensch, Vieh und potentiell auch für das ostafrikanische Küstenfieber in einer sonst unwirtlichen Gegend.

Detaillierte Informationen für den ganzen Kontinent können den Meldungen der meteorologischen Meßstationen entnommen werden. Das Centro Internacional de Agricultura Tropical (CIAT) in Kolumbien sammelte in Afrika solche Stationsmeldungen von total 5202 Stationen für den Niederschlag, von 1313 Stationen für die monatlichen Minimum- und Maximumtemperaturen sowie von 1061 Stationen für die Evaporation und stellte uns diese Daten zur Verfügung.?

Die Qualität dieser Daten abzuschätzen fiel sehr schwer, weil sie von sehr unterschiedlichen Quellen stammen und weil wenig über ihre Verarbeitung am CIAT sowie allenfalls vorgenommene Korrekturen bekannt war. Deswegen wurden die Daten jeder Station so gründlich wie möglich auf Ausreißerwerte geprüft, automatisch und visuell. Für die visuelle Kontrolle wurde ein Programm entwickelt, welches erlaubt, interaktiv auf einer Karte am Bildschirm Meßstationen auszuwählen und ihre Daten als Klimadiagramme darzustellen. Gerade die sehr aufwendige visuelle Kontrolle und das Vergleichen der dargestellten Diagramme mit denjenigen von Nachbarstationen förderte etliche Eingabefehler zutage, welche korrigiert werden konnten.

Eine automatische Interpolation von meteorologischen Daten über eine größeres Gebiet mit heterogenem Klima ist äußerst komplex und noch wenig erforscht. Die von den GIS angebotenen Interpolationsverfahren können nicht direkt verwendet werden, weil die meteorologischen Daten abhängig von weiteren Faktoren sind, beispielsweise der Höhe über Meer und der Topographie. Für eine höhenabhängige Interpolation der monatlichen Extremtemperaturen wurde deshalb ein eigenes Programm entwickelt, welches in einem Afrika bedeckenden Raster von 310 Reihen und 290 Kolonnen mit Zellen von ca. $25 \times 25 \mathrm{~km}$ Größe arbeitet. Für jede Zelle werden zunächst die fünf nächsten Meßstationen gesucht. Mit Hilfe eines globalen Höhenmodells ${ }^{8}$ werden anschließend die Höhendifferenzen zwischen der Zelle und den fünfMeßstationen ermittelt und zur Korrektur der Temperaturmeßwerte verwendet. ${ }^{9}$ Die Temperatur der jeweiligen Zelle berechnet sich dann als distanzgewichtetes Mittel der fünf höhenkorrigierten Meßwerte.
Das gleiche Programm kam für die Interpolation der monatlichen Evaporation zum Einsatz, wobei aber, wegen der signifikanten linearen Korrelation zwischen Evaporation und Temperatur in den verwendeten Daten, temperatur- und nicht mehr höhenabhängig interpoliert wurde.

Implementierbare Modelle, welche die Abhängigkeit der an Meßstationen registrierten Niederschläge von anderen Parametern beschreiben, existieren nicht für den ganzen afrikanischen Kontinent. Da jedoch Niederschlagsdaten von zahlreicheren und besser verteilten Stationen gemeldet werden als Extremtemperaturen, wurde die direkte Interpolation der Stationsdaten mit Routinen von ARC/INFO ohne Berücksichtigung von weiteren Einflüssen als vertretbar erachtet.

\subsection{Vegetation}

Indem die Vegetation ein geeignetes Mikroklima schaffen kann und Wirttiere anlockt, hat sie einen entscheidenden Einfluß auf die Entwicklung und das Überleben der braunen Zecke. Information über die heutige Vegetationsbedeckung von Afrika ist nur von Satelliten erhältlich, beispielsweise der gut erforschte Vegetations-Index NVDI (Normalized Difference Vegetation Index). Seiner hohen Korrelation mit grüner Biomasse, Niederschlag und Bodenfeuchte wegen wurde er auch in der vorliegenden Studie verwendet. ${ }^{10}$

\section{Die Eignungskartierung: Datenauswertung}

\subsection{Einfiihrung}

Waren die Grundlagedaten erhoben und eingegeben, konzentrierte sich die Arbeit auf die Bestimmung von geeigneten Lebensräumen für die braune Zecke. Dabei kann zwischen einer rein klimatischen Eignung und einer umfassenderen, die Vegetationsbedingungen miteinschließenden Eignung des Lebensraumes umterschieden werden.

\subsection{Eignung des Klimas}

Die klimatische Eignung eines bestimmten Ortes kann mit dem an der Commonwealth Science And Industrial Research Organization (CSIRO) in Australien entwikkelten CLIMEX-Modell" bestimmt werden. Es berechnet einen klimatischen Eignungsindex (Ecoclimatic Index), welcher das klimatische Potential für das Wachstum einer Zeckenpopulation und die Wahrscheinlichkeit ihres Überlebens während klimatisch ungünstigen Zeiten ausdrückt. Ferner werden vier Stress-Indizes berechnet, welche die Effekte von extremen klimatischen Bedingungen auf die Zeckenpopulation ausdrücken und erklären, warum die Zecken in bestimmten Gebieten nicht vorkommen können. 


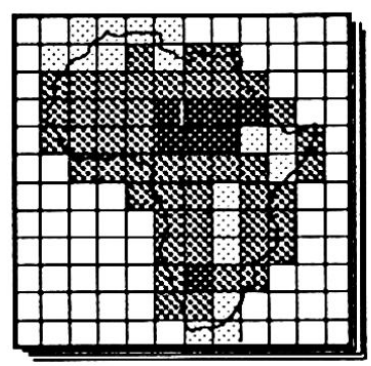

Max.

Temp.

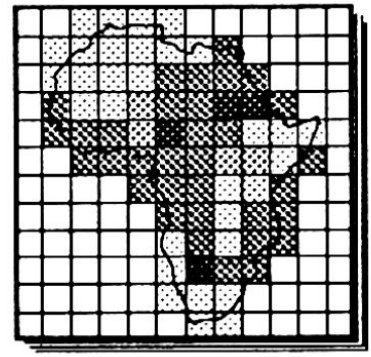

Min.

Temp.
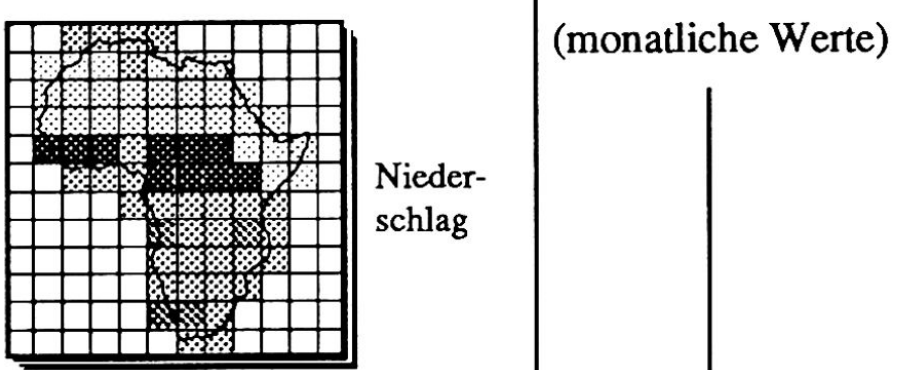

\section{Meteorologische Daten}

Nieder-

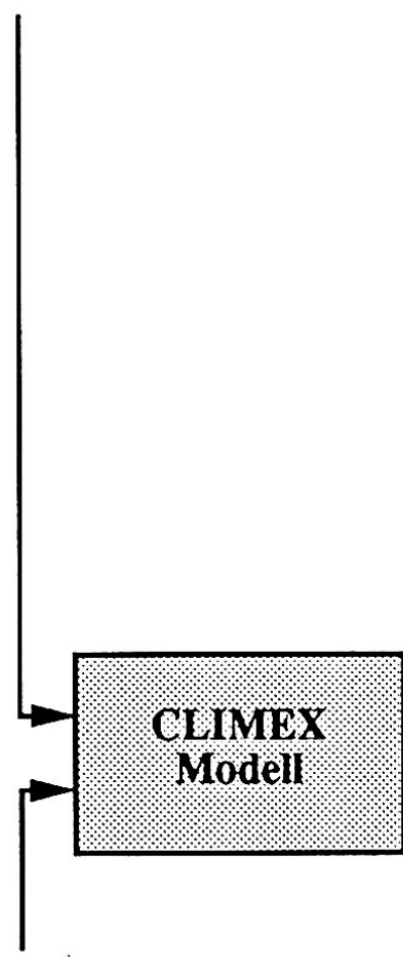

Höhe ü. M. gg. Länge gg. Breite

Evaporation
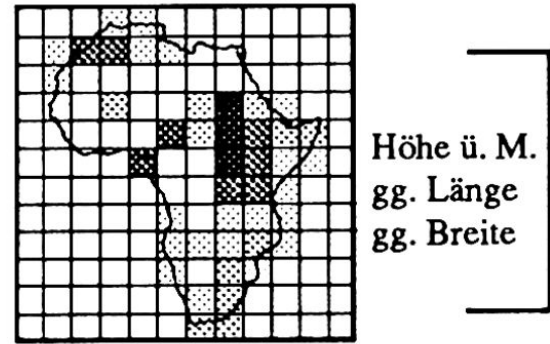

\section{Lage-Daten}

Nässestress

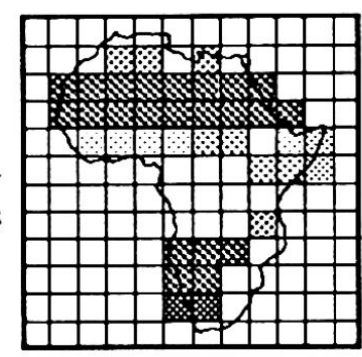

Abb. 3: Das CLIMEX-Modell 
Diese Indizes können mit dem CLIMEX-Modell für verschiedene Zeckenarten berechnet werden. Dafür müssen als meteorologische Daten die zwölf monatlichen Werte der Minimum- und Maximumtemperaturen, des Niederschlags und der Evaporation sowie die Lageinformation bestehend aus Höhe über Meer, geographischer Länge und Breite ins Modell eingegeben werden (Abbildung 3). Total ergibt das für jeden Ort 51 Eingabe-Parameter, aus welchen 5 Indizes berechnet werden.

Als Orte für die Berechnung der klimatischen Eignung dienten die bei der Interpolation der meteorologischen Daten generierten Rasterzellen. Das ursprüngliche CLIMEX-Programm von CSIRO wurde von GRID so umgestaltet, daß es große Datenmengen (43 492 Rasterzellen für den ganzen Kontinenten) aus einem rasterorientierten GIS übernehmen und die Resultate auch in einem entsprechenden Format ausschreiben kann. ${ }^{12}$.

\subsection{Eignung des Lebensraumes}

Der Schritt von der klimatischen zur Lebensraum-Eignung bedingt den Einbezug des Vegetations-Indexes in ein Modell. Obwohl die verwendeten Klima- und NDVIDaten aus nicht überlappenden Zeitabschnitten stammen, zeigen sie eine starke Korrelation bezüglich möglicher Zeckenpopulationen. Interessanter als diejenigen Gebiete, wo sowohl Klima als auch der NDVI auf günstige Bedingungen für Zecken hinweisen, sind diejenigen Gebiete, wo widersprüchliche Aussagen auftreten.

So gibt es klimatisch geeignete Gebiete, wo beispielsweise wegen Überweidung oder ungeeigneten Böden keine Vegetation vorhanden ist. Diese können als mögliche Lebensräume der braunen Zecke ausgeschlossen werden, weil die Zecke auch bei besten klimatischen Bedingungen ohne Vegetation nicht überleben kann.

Gebiete mit reichlicher Vegetation, welche gemäß CLIMEX ungeeignet sind für die Zecke, lassen sich auf zwei Arten erklären:

- Die neueren NDVI-Daten widerspiegeln klimatische Bedingungen, welche verschieden sind von den langjährigen Mittelwerten der Meßstationen. So erhielten wir Berichte von massiven Küstenfieber-Ausbrüchen in klimatisch ungeeigneten Gebieten in Zimbabwe. Genauere Untersuchungen zeigten, daß in jenen Gebieten in den letzten Jahren überdurchschnittlich viel Regen eine üppigere Vegetation ermöglichte; Rinder mit Zecken folgten nach, und das Küstenfieber stieß in ein neues Gebiet vor.

- Die reichliche Vegetation ist auf ein geeignetes Mikroklima in einem kleineren Gebiet ohne meteorologische Meßstation zurückzuführen.

Diese anscheinenden Widersprüche und ihre Erklärung zeigen, daß mit Hilfe des NDVI die aus meteorologischen Stationsdaten abgeleitete klimatische Eignung zu einer Eignung des Lebensraumes erweitert werden kann. Nach einer sorgfältigen visuellen Interpretation der NDVI-Daten wurden zwei Schwellenwerte festgelegt. Unter einem NDVI von 0,15 wird ein Ort, unabhängig von seiner klimatischen Eignung, als für die braune Zekke ungeeignet bezeichnet. Klimatisch ungeeignete Orte mit einem NDVI von mehr als 0,25 hingegen wurden als «geeignet dank günstigem Mikroklima» bezeichnet.

\section{Veterinär-medizinische Resultate}

Wie erwartet sind die traditionellen Küstenfieber-Gebiete um den Viktoria-See und im östlichen Zaire bezüglich Lebensraum und Klima (Abbildungen 4a und b) für die braune Zecke sehr geeignet. Zudem wurde jedoch festgestellt, daß größere, bis jetzt noch küstenfieberfreie Gebiete in Äthiopien, Zentral- und West-Afrika ebenfalls geeignete Lebensräume für die braune Zecke wären. Die Rinderverteilungskarte zeigt, daß in diesen Gebieten auch große Viehbestände vorhanden sind, was bei einem Einschleppen der Zecke eine rasche Verbreitung der Krankheit begünstigen würde.

Während die Ausbreitung des Fiebers gegen Westen durch die Viehverschiebungen hemmenden tropischen Regenwälder Zentralafrikas verhindert wird, verunmöglichen klimatische Gegebenheiten seine Ausbreitung nach Äthiopien. Ein Vergleich der Karten der klimatischen Eignung und des Hitzestresses (Abbildungen 5a und b) zeigt deutlich die wegen großer Hitze ungeeigneten Gebiete im nördlichen Kenia. Diese sind mehr als 3 Tagesreisen der dort ansässigen Nomaden mit ihren Herden breit. Die Zecke, welche nur 3 Tage auf dem Rind bleibt und sich dann vollgesogen fallen läßt, fällt somit unweigerlich in ein für sie ungeeignetes Gebiet und stirbt dort wegen der großen Hitze. Damit bilden diese heißen Gebiete eine natürliche Barriere gegen die Ausbreitung des ostafrikanischen Küstenfiebers in Richtung Norden.

\section{Schlußbemerkungen und Ausblick}

\subsection{Die Bedeutung eines GIS in Ausbreitungs- untersuchungen von Krankheiten}

Der Aufbau des GIS mit epidemiologischen Parametern des ostafrikanischen Küstenfiebers sowie Klimadaten nahm ungefähr anderthalb Jahre in Anspruch und lastete während dieser Zeit je einen Experten von ILRAD und GRID beinahe vollständig aus. Weitere Personen halfen bei der Datenaufnahme oder stellten ihr Fachwissen zur Verfügung.

Die Verschiedenheit der Datenquellen machte es unmöglich, eine Datenbank von homogener Qualität aufzubauen. Sehr oft standen wir vor der Wahl, entweder für den ganzen Kontinent oder Teile davon ungenaue Daten zu akzeptieren oder einen Parameter ganz fallenzulassen. LESSARD ${ }^{13}$ verlangt deshalb ein standardisiertes Meldesystem für das Küstenfieber, wobei im Hinblick auf eine spätere Verwendung in einem GIS bereits bei der $\mathrm{Da}$ tenerhebung allen Beobachtungen möglichst genaue Koordinaten zugeordnet werden sollten. 


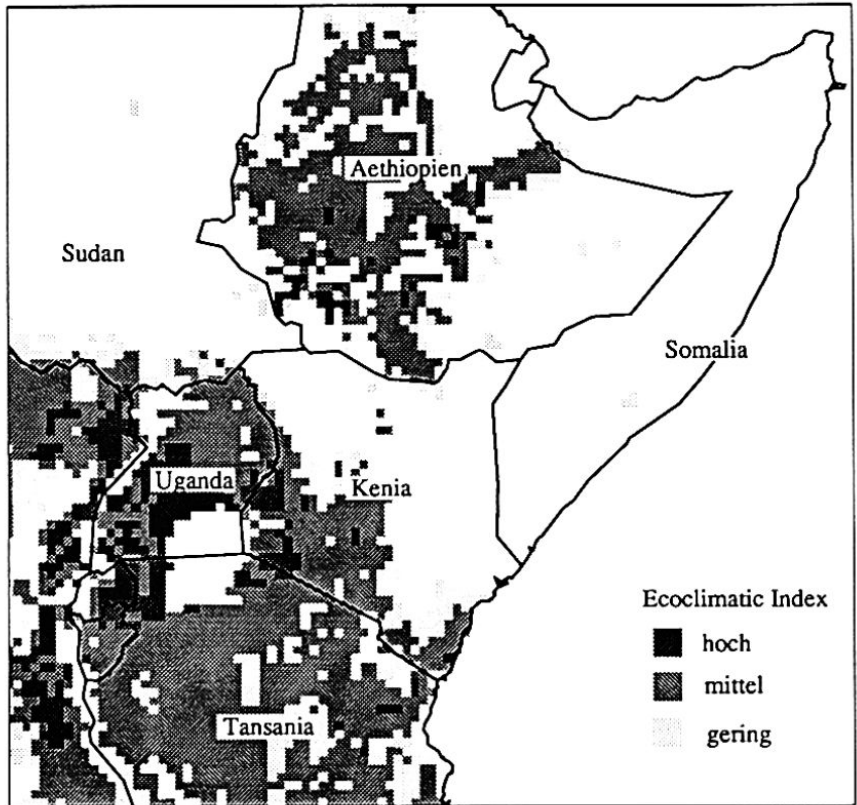

Abb. 4a: Ecoclimatic-Index

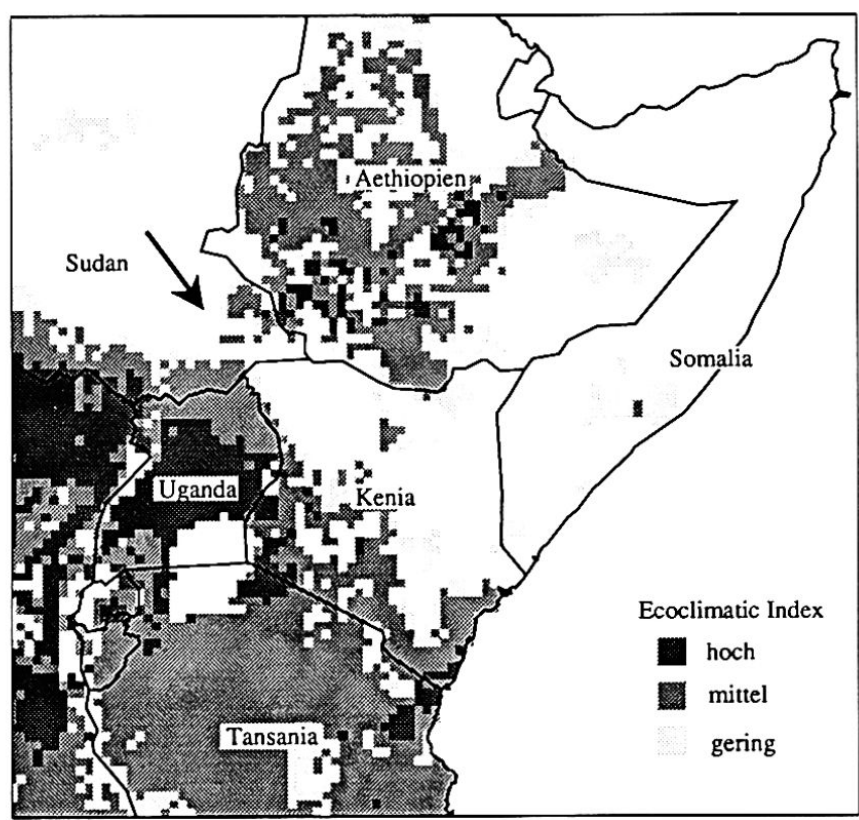

Abb. 5: Simulation max. Temp. $-2^{\circ} \mathrm{C}$ a) Ecoclimatic-Index

Trotz der teilweise bescheidenen Qualität der eingegebenen Daten erwies sich das GIS in der vorliegenden Untersuchung als ein äußerst nützliches, entscheidungsunterstützendes Werkzeug für Fragen der Krankheitsbekämpfung und -vorbeugung. Denn dank dem GIS-Einsatz konnten erstmals Eignungskarten für ganz Afrika erstellt werden, und erst ihr Vergleich mit der Küstenfieber- und Rinderverteilungskarte erlaubte die Identifikation von möglichen neuen Ausbreitungsgebieten sowie von besonders gefährdeten, bereits befallenen Gebieten. Nur durch ihre Identifikation können solche Gebiete vor einem Befall beschützt werden bzw. können die beschränkt zur Verfügung stehenden Mittel zur Bekämpfung der Krankheit gezielt eingesetzt werden. In diesem Sinne wurden die Resultate dieser Untersuchung in einer Kar-

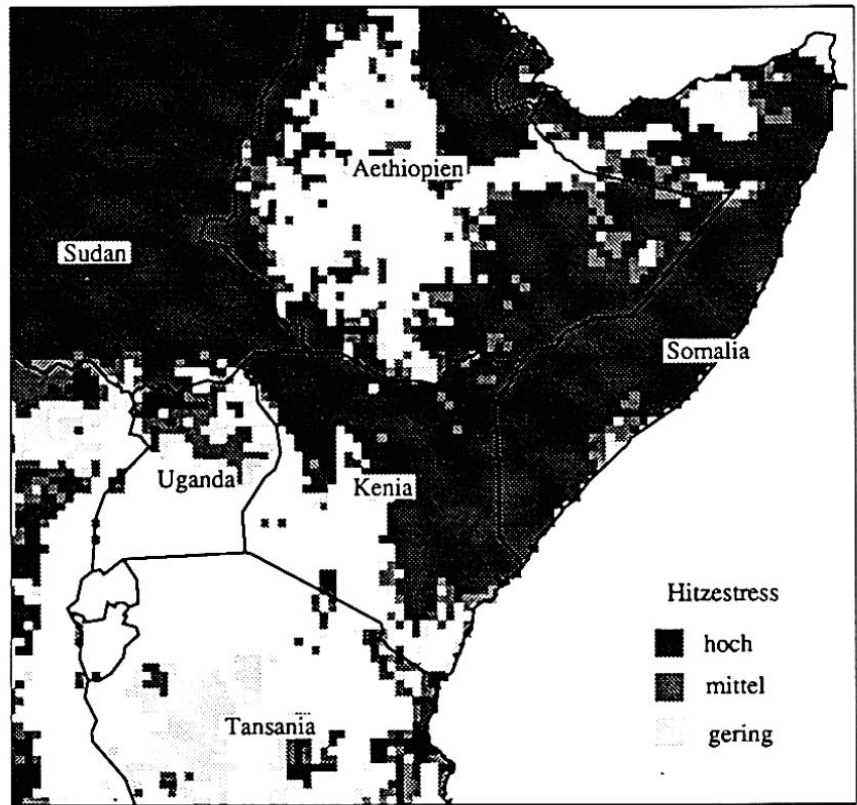

Abb. 4b: Hitzestress

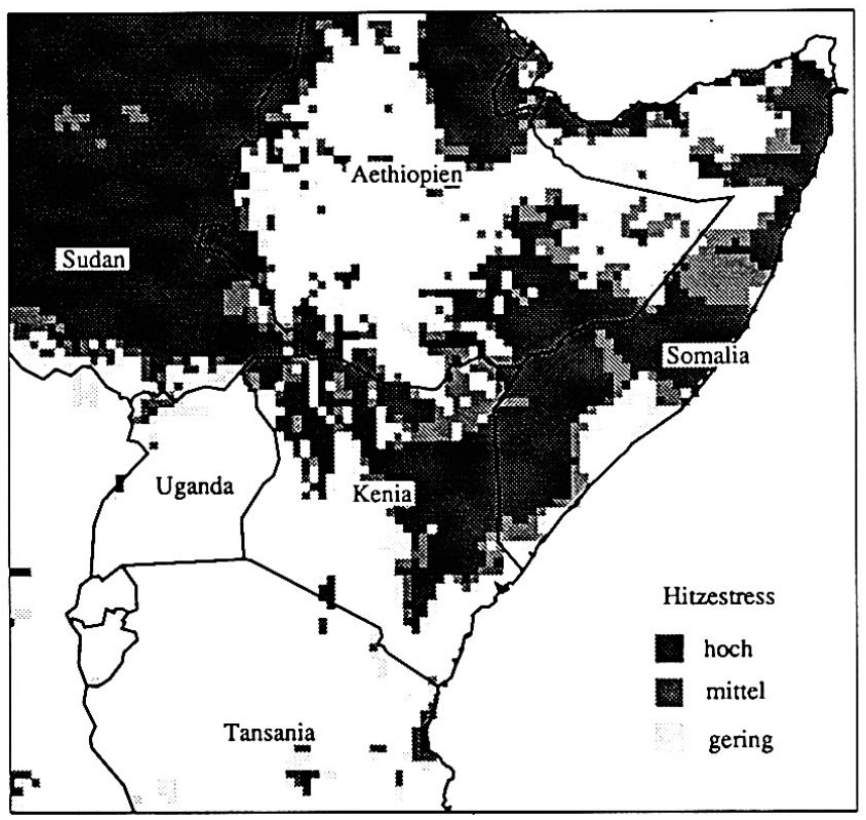

Abb. 5: Simulation max. Temp. $-2^{\circ} \mathrm{C}$ b) Hitzestress

tenserie dargestellt und interessierten Stellen zugänglich gemacht.

\subsection{Klimadaten}

Die Interpolation von Klimadaten und deren Einbezug in ein GIS stand zwar nie im Vordergrund der vorliegenden Untersuchung, verursachte aber einen beträchtlichen Aufwand, da keine interpolierten Klimadaten von genügender räumlicher Auflösung zur Verfügung standen. Eine Bewertung der Qualität der interpolierten Daten erwies sich als äußerst schwierig. Die mehrheitliche Übereinstimmung der mit CLIMEX berechneten Eignungskarten mit der bekannten Verteilung der braunen 
Zecke weist aber darauf hin, daß die verwendeten Stationsdaten und die angewandten Interpolationsmethoden für diese Untersuchung genügten. So hatten erst später entdeckte Inkonsistenzen in niederschlagsarmen Gebieten keinen Einfluß auf die Modellrechnung, weil die dortigen Niederschläge für ein Überleben der braunen Zecke ohnehin zu klein waren.

Wegen der großen Nachfrage von UNEP-internen und -externen Projekten nach Klimadaten ist GRID momentan damit beschäftigt, zuverlässige Stationsdaten zu erwerben und die angewandten Interpolationsmethoden zu überprüfen und anzupassen.

\subsection{Von Simulationen zur ständigen Überwachung}

Mit dem von GRID modifizierten CLIMEX-Programm können auch Variationen des Klimas simuliert und ihre Auswirkungen auf die Verbreitung des ostafrikanischen Küstenfiebers bestimmt werden. Neue, unter den simulierten Bedingungen für die braune Zecke potentiell geeignete Gebiete lassen sich dadurch sofort identifizieren. So wurde untersucht, unter welchen klimatischen Bedingungen sich das ostafrikanische Küstenfieber nach Äthiopien ausbreiten könnte. Das wäre erst bei einer wenig wahrscheinlichen Verminderung der monatlichen Maximaltemperaturen um 2 Grad Celsius der Fall. Die Abbildungen 5a und $\mathrm{b}$ zeigen, wie die natürliche Barriere der wegen Hitze ungeeigneten Gebiete im südlichen Sudan so stark schrumpfen würde, daß sie innert weniger als drei Tagen überwunden werden könnte. Ein eher wahrscheinlicher Temperaturanstieg hingegen würde die Barriere noch verstärken, während die Barriere auf Variationen des Niederschlages praktisch nicht reagiert. Demnach besteht für Äthiopien mit größter Wahrscheinlichkeit keine Gefahr, vom ostafrikanischen Küstenfieber heimgesucht zu werden.

Gebiete hingegen, welche wegen veränderter klimatischen geeignet werden, müssen rechtzeitig gewarnt werden. Es wird daher von GRID angestrebt, in regelmäßigen Zeitabschnitten aktuelle Klimadaten zu erwerben, sie zu interpolieren und in das aufgebaute GIS einzufügen, womit dieses zu einem Frühwarnsystem ausgebaut werden kann.

\subsection{Die Visualisierung der Untersuchung}

Durch die Visualisierung wird ein besseres Verständnis von komplexen Sachverhalten und Zusammenhängen erreicht. Die graphischen Ausgabemöglichkeiten des verwendeten GIS wurden deshalb von GRID zur Visualisierung des ganzen Küstenfieber-Projektes in einer leicht verständlichen Computer-Präsentation eingesetzt. Erst die darin enthaltenen Karten und Diagramme machten unsere Untersuchungen einem breiteren Publikum zugänglich.

Die positiven Reaktionen vieler Besucherinnen und Besucher der UNEP, welche anhand dieser Präsentation einen Einblick in das Küstenfieber-Projekt gewannen, zeigten einerseits ein großes Interesse an Klimadaten und andererseits, daß Untersuchungen der räumlichen Aspekte von anderen, durch Vektoren auch auf Menschen übertragenen Krankheiten (Malaria, Bilharziose oder Schlafkrankheit) bisher häufig vernachlässigt wurden, weil dafür geeignete Methoden und Werkzeuge noch nicht existieren oder bekannt waren. Mit Geographischen Informationssystemen existiert jedoch ein solches Werkzeug, welches hoffentlich - auch dank der GRID-Präsentation über das Küstenfieberprojekt - über die Geographie hinaus bekannter werden wird.

\section{Anmerkungen}

1 Verwendet wurde ARC/INFO auf einer Micro VAX 3600.
2 Kundert, 1989
${ }^{3}$ z. B. Lessard et al., 1989

${ }^{4}$ Alle Karten werden anschließend in die von GRID für seine Afrika-Datenbank verwendeten "Miller Oblated Stereographic Projection" transformiert.

${ }^{5}$ Adeniji, 1988

6 The African Elephant Database Project, Burrill and Douglas-Hamilton, 1987

7 CIAT, Jones 1988

8 Verwendet wurde das Höhenmodell des USGS (United States Geological Surveys) mit 10 Minuten Auflösung. .

9 In dieser Untersuchung wurde für den ganzen Kontinent eine Temperaturveränderung von 1,0 Grad Celsius pro 100 Meter Höhendifferenz angenommen. Vgl. Heyer 1979.

10 Der NDVI stammt vom AVHRR (Advanced Very High ResoIution Radiometer) des NOAA-Staelliten. In dieser Untersuchung wurden die monatlichen NDVI-Maximalwerte des Jahres 1987 verwendet. Neuere Daten waren in Nairobi nicht erhältlich.

11 CSIRO, 1985

12 Diese Daten können vektorisiert und in ARC/INFO zusammen mit anderen GRID-Dateien weiterverwendet werden.

13 Lessard et al., 1989

\section{Literatur}

ADENIJI, K. O. (1988): Distribution Of Cattle In Africa, Third Edition OAU/STRC, Nairobi.

BURRILL, A. \& DOUGLAS-HAMILTON, I. (1987): African Elephant Database, Nairobi.

CSIRO (1985) User's Guide To CLIMEX, A Computer Program For Comparing Climates In Ecology. In: CSIRO Aust. Div. Rep. No. 35, 1-29.

HEYER, J. (1979): Witterung und Klima, 5. Auflage, BSG BG Teubner Verlagsgesellschaft, Leipzig.

JONES, P. (1988): Climate Database, V 3.04, Magnetband, CIAT, Cali, Kolumbien.

KUNDERT, K. (1989): Isolating East Coast Fever High Risk Areas. In: ARC/INFO, The Forth Annual ESRI European User Conference, Rome.

LESSARD, P. et al. (1989) Geographic Information Systems For Studying The Epidemiology Of East Coast Fever. Manuskript ILRAD, zur Veröffentlichung in The Veterinary Record. 